\title{
Disease Prediction Chatbot
}

\section{Shraddha A. S ${ }^{1}$, Shreepada Bhat ${ }^{1}$, Shubhashri V. K ${ }^{1}$, Sinchana Karnik ${ }^{1}$, Narender M $^{2}$}

1Department of Computer Science and Engineering at National Institute of Engineering Manandvadi Road, Mysuru, Karnataka, India

${ }^{2}$ Assistant Professor, Department of Computer Science and Engineering at National Institute of Engineering Manandvadi Road, Mysuru, Karnataka, India

\section{ABSTRACT}

\section{Article Info}

Volume 7, Issue 3

Page Number: 632-636

Publication Issue :

May-June-2021

\section{Article History}

Accepted : 20 June 2021

Published : 30 June 2021
Applications in the field of machine learning and artificial intelligence have been in great demand over the recent decade. Now it has various applications in the field of health industry. With the help of machine learning algorithm prediction of diseases has been made easier. Now doctors can concentrate only on treatment with the help of technology. Technology is accelerating innovations in the healthcare domain which has increased people's standard of living over the years. Here in our project we are making a healthcare chatbot with help of Natural language processing and machine learning algorithm to predict disease. User interacts with the chatbot just like one interacts with his doctor and based on the symptoms provided by users and the chatbot will identify the symptom and predict the disease.

Keywords: Chatbot, NLP, Naïve bayes

\section{INTRODUCTION}

It is important to maintain health if one wishes to be happy. Only a healthy body can have a healthy mind. Nowadays, people are less aware of their health. In their busy life, they forget to take suitable measures to maintain their health and are less aware of their health status.

Such a problem can be avoided by using the symptoms driven disease prediction application. This research intends to apply the concepts of natural language processing and machine learning to create a chatbot application. People can interact with the chatbot just like they do with another human and through a series of queries; chatbot will identify the symptoms of the user and thereby, predicts the disease using a machine learning algorithm. This system can be of great use to people in conducting daily check- ups, makes people aware of their health status and encourages people to make proper measures to remain healthy. According to this research, such a system is not widely used and people are less aware of it. Executing this proposed framework can help people avoid the timeconsuming method of visiting hospitals by using this free of cost application, wherever they are.

The disease prediction chatbot can make a great change in the health of our society. It is more reliable and less prone to human errors. People avoid visiting

Copyright: @ the author(s), publisher and licensee Technoscience Academy. This is an open-access article distributed under the terms of the Creative Commons Attribution Non-Commercial License, which permits unrestricted non-commercial use, distribution, and reproduction in any medium, provided the original work is properly cited 
hospitals over small issues which can be major problem in the future. Idea is to focus on the solution which is free of cost and available throughout the day. In this way people can be more aware about their health. A user can ask questions at any time of the day even with his busy schedule and keep a check on his own health without visiting any specialized doctor just for consultation.

\section{LITERATURE SURVEY}

Advancement in technology has a far-reaching effect in the field of Healthcare. Technology has not only helped the doctors but also has provided a first-hand testing set for the patients

[1] Chatbot for Disease Prediction and Treatment Recommendation using Machine Learning Published in 2019 3rd International Conference on Trends in Electronics and Informatics (ICOEI) : The proposed system is to create an alternative to this conventional method of visiting a hospital and making an appointment with a doctor to get diagnosis. People can interact with the Chatbot just like they do with another human and through a series of queries with the chatbot. The chatbot will identify the symptoms of the user and thereby, predicts the disease and recommends treatment.

[2] Medbot: Conversational Artificial Intelligence Powered Chatbot for Delivering Tele-Health after COVID-19- Published in 2020 5th International Conference on Communication and ElectronicsSystems (ICCES):Telemedicine can be used by medical practitioners to connect with their patients during the recent Corona virus outbreak, whilst attempting to reduce COVID-19 transmission among patients and clinicians. Amidst the pandemic, Telemedicine has the potential to help by permitting patients to receive supportive care without having to physically visit a hospital by using a conversational artificial intelligence-based application for their treatment. Thus, tele health will rapidly and radically transform inperson care to remote consultation of patients. Because of this, it developed a Multilingual Conversational Bot based on Natural Language Processing (NLP) to provide free primary healthcare education, information, and advice to chronic patients. The study introduces a novel computer application acting as a personal virtual doctor that has been opportunely designed and extensively trained to interact with patients like human beings. This application is based upon a server-less architecture and it aggregates the services of a doctor by providing preventive measures, home remedies, interactive counseling sessions, healthcare tips, and symptoms covering the most prevalent diseases in rural India. The paper proposes a conversational bot "Aapka Chikitsak" on Google Cloud Platform (GCP) for delivering telehealth in India to increase the patient's access to healthcare knowledge and leverage the potentials of artificial intelligence to bridge the gap of demand and supply of human healthcare providers. This conversational application has resulted in reducing the barriers for access to healthcare facilities and procures intelligent consultations remotely to allow timely care and quality treatment, thereby effectively assisting the society.

[3] A Medical ChatBot-Published in June 2018 International Journal of Computer Trends and Technology : Normally Users are not aware about all the treatment or symptoms regarding the particular disease. For small problems users have to go personally to the hospital for check-up which is more time consuming. Also handling the telephonic calls for the complaints is quite hectic. Such a problem can be solved by using medical ChatBot by giving proper guidance regarding healthy living. The medical chatbots functioning depends on Natural language processing that helps users to submit their problem about the health. The User can ask any personal query related to health care through the chatbot without physically available to the hospital. By Using 
Google API for voice-text and text voice conversion. Query is sent to ChatBot and gets a related answer and display answer on android app. The System's major concern behind developing this web based platform is analysing customer's sentiments

[4] In recent times, healthcare is becoming more accessible to a wider group of people through the medium of technology. The concepts of artificial intelligence, machine learning and neural networks have provided substantial assistance in the field of healthcare. In today's fast-paced world, people tend to neglect their health which may result in a critical problem. Such a problem can be avoided by using the symptoms driven disease prediction application. Our project focuses on providing the users immediate and accurate prediction of the diseases based on their symptoms along with a detailed analysis of their pathology reports. The disease prediction chatbot is developed using natural language processing and machine learning algorithms. For the prediction of diseases, we have used two classification algorithms namely, Decision tree and KNN (k-nearest neighbors). The performance of these techniques are compared and based on their accuracy, the best model is selected. As per our results, the accuracy of Decision Tree and $\mathrm{KNN}$ are $92.6 \%$ and $95.74 \%$ respectively. This project also looks forward to providing medical consultation on the predicted disease. The pathology report analysis is performed using the concept of Optical Character Recognition (OCR). Tesseract is an open-source recognition engine to perform OCR. The text extracted from the report is used for interpreting the results in an easier way and to provide a graphical analysis of the test results.

\section{PROBLEM STATEMENT}

Hospitals are the most widely used means by which a sick person gets medical check-ups, disease diagnosis and treatment recommendation. This has been a practice by almost all the people over the world.
People consider it as the most reliable means to check their health status. Normally users are not aware about all the treatment or symptoms regarding the particular disease. For small problems user have to go personally to the hospital for check-up which is more time consuming. Consequently, prediction system using Machine Learning has recently been a significant effort to lessen doctor's workload and develop the overall competence of the health care method with machine learning.

\section{PROPOSED SYSTEM}

In this proposed system, a medical chatbot is built that motivates users to discuss their health problems as shown. Based on the symptoms provided by the users. Chat bot will predict the disease. This chat bot system will be able to identify symptoms from user interaction. Using these extracted symptoms, chat bot predicts the disease and recommends treatment. By this way Chatbot becomes very useful to the people who focus on their wellbeing. The user can interact with chat bot and can depend on them to gets a timely treatment. The chatbot allows users to login to the system. Here User registers on chatbot application. User then interacts with the system and the words, symptoms are recognized by the use of natural language processing, and the disease is predicted using a naive bayes algorithm. There is an admin who controls the chat bot application. The admin views the details of all the users and can even add, delete or update symptoms and diseases. The chatbot is trained on symptoms-disease dataset. Fig.1 shows a model where in the symptoms identified by the user, the system recognizes the disease. The chatbot encourages patients to discuss their medical issues and predict the disease.

Advantages of Proposed System : 
- Can efficiently predict the disease based on symptoms.

- Provides diet plans according to the predicted disease.

- One can view the hospital nearby to his location in case he needs to take any health related test based on the location he chooses

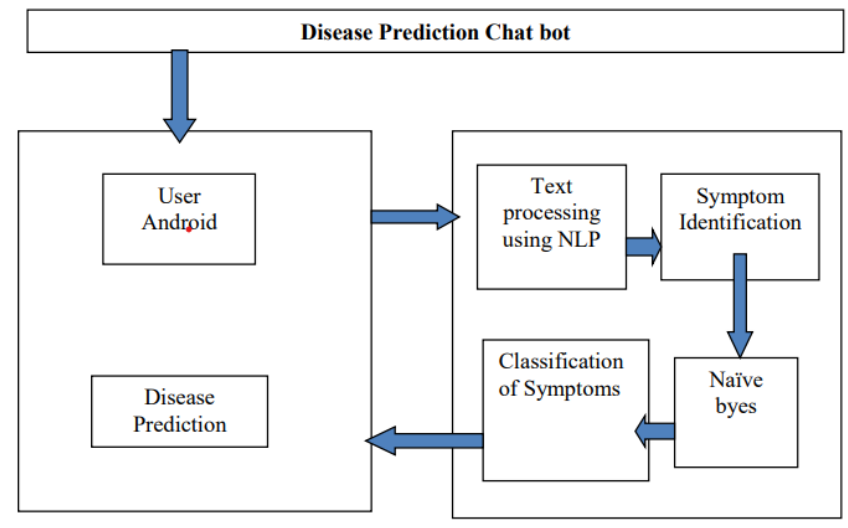

Figure 1.Working model of proposed system

Natural language processing (NLP) is a field of artificial intelligence that helps in designing a program to process and analyse natural language data. It permits to set up communications among PCs and people in a characteristic language. The proposed framework is a talk interface that depends on Retrieval based model of NLP where the bot is prepared with a lot of inquiries with a set Such a wise chatbot can manage the patients by comprehension and surveying their side effects that they are features of the Proposed System. Proposed system is an Android Application that has a Chatbot in it. The working of the system is as follows:

- Build a simple and interactive real time chat system.

- Dedicated system, which is able to solve all the queries regarding a medicine.

- Effective Symptom based disease prediction.

- $\quad$ Suggest nearby hospitals based on the disease.

\section{SYSTEM ARCHITECTURE}

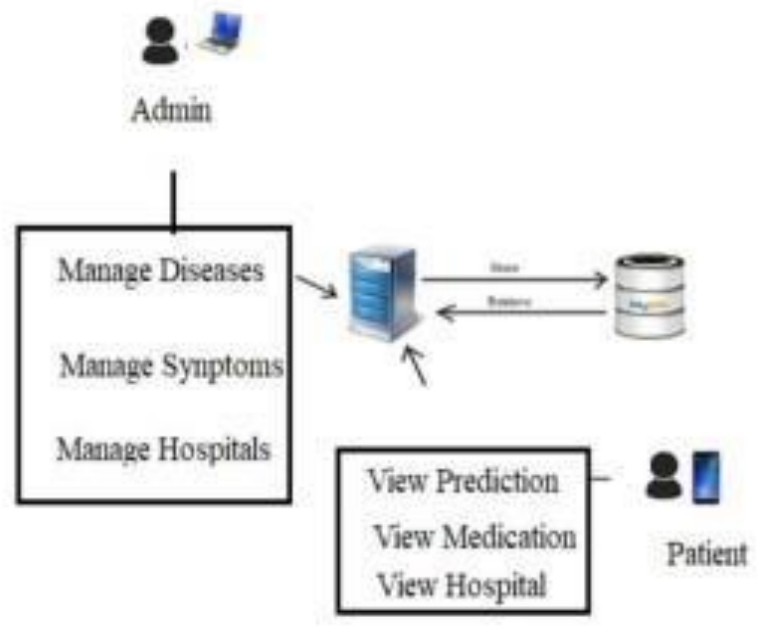

Figure 2. System Architecture diagram

\section{DATA FLOW DIAGRAM}

Fig. 3 shows an Admin who manages disease, symptoms and hospitals.

Database is used to store and retrieve the information.

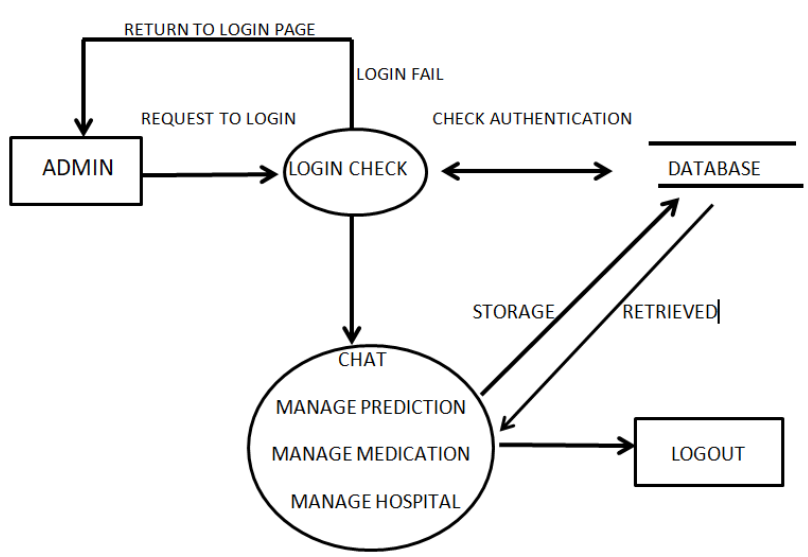

Figure 3. Admin flow diagram

Fig.4 shows a Patient flow where one can view the predicted disease, medication required and nearby hospitals according to the prediction. 


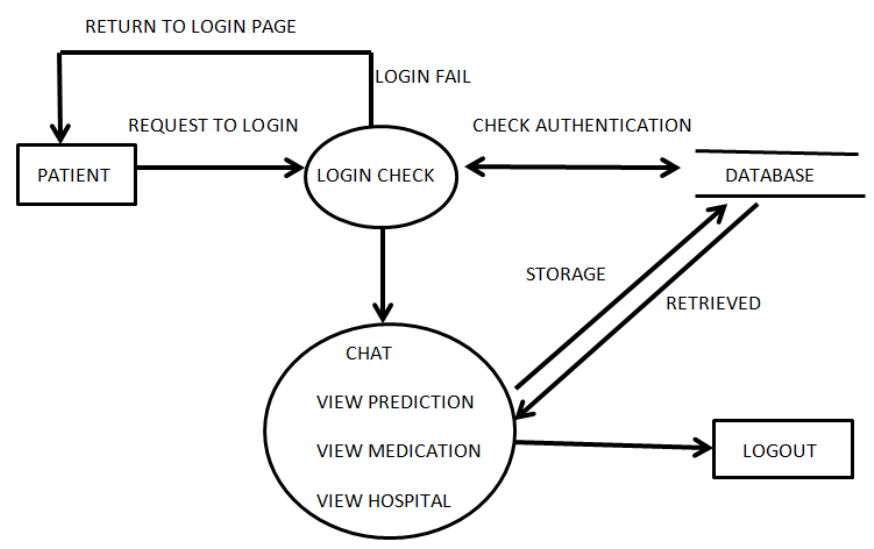

Figure 4. Patient flow diagram

\section{CONCLUSION}

Our project aims to be a medical chat bot which can be used to replace the conventional method of disease diagnosis and treatment recommendation. Chat bot can act as a doctor. The chatbot acts as a user application. The user of this application can specify their symptoms to the chat bot and in turn, chat bot will specify the health measures to be taken. General information about symptoms and diseases are available in the dataset and thus the chat bot instance can provide information about disease and treatment to the user. After analysing the symptoms of the different users, it finally predicts the disease to the user and provides a link where details about the treatment are visible. We believe this approach incorporated into existing strategies in the field of healthcare will provide assistance to the healthcare specialist and patients.

\section{REFERENCES}

[1]. Rohit Binu Mathew ,Sandra Varghese, Sera Elsa Joy and Swanthana Susan Alex. Chat bot for Disease Prediction and Treatment Recommendation using Machine Learning. 2019 3rd International Conference on Trends in Electronics and Informatics (ICOEI).IEEE.

[2]. Divya Madhu, Jain C, J Neeraj, Elmy Sebastain, Shinoy Shaji and Anandhu Ajaykumar, "A
Novel Approach for Medical Assistance Using Trained Chatbot", International Conference on Inventive Communication and Computational Technologies(ICICCT 2017).

[3]. Rashmi Dharwadkar and Neeta A. Deshpande, "A Medical ChatBot", International Journal of Computer Trends and Technology (IJCTT), vol. 60, no. 1, pp. 41-45, June 2018.

[4]. Hiba Hussain, Komal Aswani, Mahima Gupta, Dr. G.T.Thampi. Implementation of Disease Prediction Chatbot and Report Analyzer using the Concepts of NLP, Machine Learning and OCR, International Research Journal of Engineering and Technology (IRJET)

\section{Cite this article as :}

Shraddha A. S, Shreepada Bhat, Shubhashri V. K, Sinchana Karnik, Narender M, "Disease Prediction Chatbot", International Journal of Scientific Research in Computer Science, Engineering and Information Technology (IJSRCSEIT), ISSN : 2456-3307, Volume 7 Issue 3, pp. 632-636, May-June 2021. Available at doi : https://doi.org/10.32628/CSEIT2173172

Journal URL : https://ijsrcseit.com/CSEIT2173172 